\title{
FORMULACIÓN Y CINÉTICA DE LA DEGRADACIÓN DE ANTOCIANINAS DEL NÉCTAR DE ZARZAMORA SILVESTRE SP. POR TRATAMIENTO
}

\section{FORMULATION AND KINETICS DEGRADATION OF ANTHOCYANINS NECTAR WILD BLACKBERRY SP. FOR TREATMENT}

\author{
Nora Veliz S., Clara Espinoza S., Miguel Quispe S. \\ Facultad de Ingeniería en Industrias Alimentarias, Facultad de Ciencias Aplicadas Universidad Nacional del Centro \\ del Perú, Perú.
}

\section{RESUMEN}

El presente trabajo de investigación tiene por objetivo formular y evaluar la cinética de degradación de antocianinas manoméricas (tacys) del néctar de zarzamora silvestre sp. por tratamiento térmico a partir de dos estados de madurez del fruto para lo cual se trabajó con pulpa en zarzamora en estado pintón y maduro, a los que se estandarizo con agua en una dilución 1:2 ajustando el pH a 3.5 y $13^{\circ}$ Brix. El producto fue pasteurizado a temperaturas de $70^{\circ} \mathrm{C}$, $80^{\circ} \mathrm{C}$ y $90^{\circ} \mathrm{C}$ por espacio de 5 y 10 minutos. Luego se procedió a la cuantificación de antocianinas monoméricas a medida que se incrementa el tiempo y la temperatura disminuye siguiendo una cinética de degradación de forma lineal $\left(r^{2}=0.8543\right)$ para el néctar con pulpa de zarzamora madura $(M)$ y forma polinómica $\left(r^{2}=0.9065\right)$ para el néctar zarzamora fisiológicamente maduro (FM). Así mismo se ha encontrado una correlación positiva entre la capacidad antioxidante y tacys $(r=0.795)$ para el néctar con pulpa $(F M)$ y una correlación positiva significativa $(r=$ $0.831^{\star}$ ) entre la capacidad antioxidante y tacys para el néctar con pulpa (M). Por último el producto fue sometido a una evaluación sensorial encontrándose que en néctar a partir de la pulpa FM no existe diferencia significativa $(p=0.314)$ mientras que en el néctar a partir de la pulpa M existe diferencia significativa $(p<0.05)$ en cuanto a su apariencia general.

Palabras claves: néctar, antocianinas, cinética de degradación.

\section{ABSTRACT}

This research aims to develop and evaluate the degradation kinetics of anthocyanins manoméricas (tacys) the nectar of wild blackberry sp. by heat treatment from two stages of maturity of the fruit for which he worked with in ripening fruit pulp blackberry and ripe, to which I had standardized with water at a 2:2 dilution adjusted to $\mathrm{pH}$ 3.5 and $13^{\circ}$ Brix. The product was pasteurized at temperatures of $70^{\circ} \mathrm{C}, 80^{\circ} \mathrm{C}$ and $90^{\circ} \mathrm{C}$ for 5 to 10 minutes. Then he proceeded to quantification of monomeric anthocyanins as time increases and the temperature decreases following degradation kinetics linearly $\left(r^{2}=0.8543\right)$ for nectar with pulp of ripe blackberry $(\mathrm{M})$ and is polynomial $\left(r^{2}\right.$ $=0.9065$ ) for nectar blackberry physiologically mature (FM). Also found a positive correlation between antioxidant capacity and tacys $(r=0.795)$ for nectar with pulp (FM) and a significant positive correlation $\left(r=0.831^{\star}\right)$ between the antioxidant capacity and tacys for nectar with pulp (M). Finally the product was subjected to sensory evaluation was that in nectar from the FM pulp there is no significant difference $(p=0.314)$ while on the nectar from the pulp $M$ there is significant difference $(p<0.05)$ as its general appearance.

Keywords: nectar, anthocyanins, degradation kinetics 


\section{INTRODUCCION}

En la actualidad los alimentos funcionales se presentan como una posible solución a la actual carencia nutricional, ocasionada por una pobre alimentación o por una mala alimentación y los efectos benéficos que presentan en la salud. Existen muchas variedades de frutas que se consumen directamente o como jugo y néctares encontrando que muchas de éstas poseen compuestos bioactivos.

El consumo de néctares a partir frutas tiene una gran demanda los mismos que pueden prepararse a partir de zarzamora silvestre aportando compuestos fenólicos, vitaminas, antocianinas, y carotenoides. Desde el punto de vista tecnológico una de las principales ventajas de las antocianinas es su hidrosolubilidad, lo cual facilita su incorporación en diferentes alimentos como néctares no obstante en la tecnología de néctares estos se pasteurizan a fin de prolongar la vida útil, reducir la actividad enzimática, la temperatura de pasteurización provoca la degradación de azucares, antocianinas y vitaminas los cuales pueden afectar significativamente las propiedades sensoriales y funcionales del producto. La presencia de estos compuestos bioactivos juega un papel importante en la prevención de enfermedades relacionadas con el estrés oxidativo, fundamentalmente a través del aporte de compuestos bioactivos de origen vegetal.

\section{MATERIALES Y METODOS}

- Tipo de Investigación: Aplicada

- Nivel de Investigación: Experimental

- Lugar de ejecución: Laboratorios de ciencia y tecnología de la FAllA y FACAP de la UNCP.

- Materia prima: Se obtuvo pulpa de mora silvestre sp. en estado pintón y maduro, para ello se recolectaron frutos provenientes del distrito de Tintay Punco Zona del VRAE - Provincia de Tayacajá - Región Huancavelica (Ubicado entre 2245 a 2350 msnm, a una Latitud Sur S: $12^{\circ} 08^{\prime} 696 "$, longitud Oeste: $\left.74^{\circ} 32^{\prime} 821\right)$.

- Análisis fisicoquímico de pulpa de mora silvestre sp. y producto terminado.

- Acidez: Porcentaje de acidez, expresado como porcentaje de ácido málico NTC 4106(1997) para mora de castilla
- pH: Método potenciométrico recomendado por AOAC (1997).

- Sólidos solubles: Método refractometrico AOAC (1995)

- Índice de madurez: Según NTC 4106(1997) ( ${ }^{\circ B r i x / ~}$ acidez)

- Análisis químico proximal: Proteína, Grasa, Humedad,, Ceniza, Fibra Cruda y

- Carbohidratos: Método, (AOAC, 1995).

- Análisis de antioxidantes de la pulpa y néctar de mora silvestre $\mathrm{sp}$.

- Determinación de antocianinas monoméricas totales:Método del pH diferencial propuesto por Fuleki y Francis (1968) modificado por Giusti y Wrolstad (2001)

- Capacidad antioxidante: DPPH.- Método basado en la reducción del radical libre estable 2,2, difenil1-picrilhidrazil (DPPH), en base húmeda metodología propuesta por Brand Williams et al. (1995) con algunas modificaciones (Rojano et al., 2008).

- Evaluación sensorial: Recomendado por Anzaldua (1998)

Metodología para la elaboración del néctar a partir de la pulpa de zarzamora sp.

Se seguirá el siguiente diagrama de flujo figura 1:

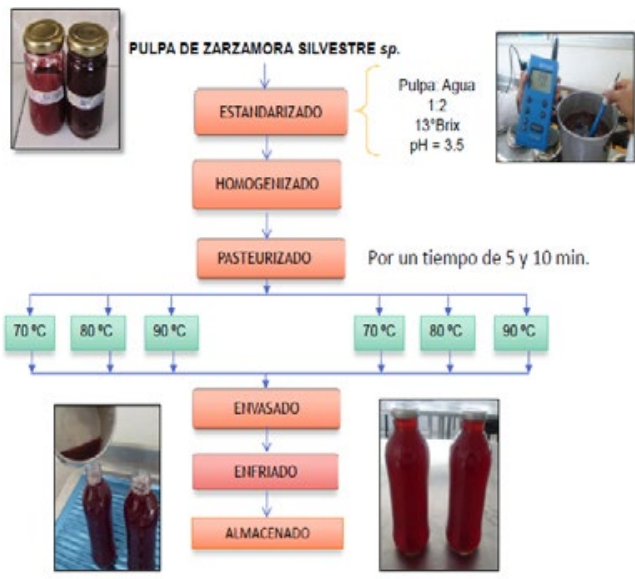

Figura 1: Diagrama flujo de proceso de la obtención del néctar de zarzamora silvestre sp. 


\section{RESULTADOS}

Tabla 1.

Análisis químico proximal de la pulpa de zarzamora silvestre $\mathrm{sp}$

\begin{tabular}{|c|c|c|}
\hline \multirow{2}{*}{$\begin{array}{c}\text { Composición } \\
\mathbf{g} / \mathbf{1 0 0} \mathbf{g}\end{array}$} & \multicolumn{2}{|c|}{ Estados de madurez de la zarzamora } \\
\cline { 2 - 3 } & Pintón & Maduro \\
\hline Humedad & $79.93 \pm 1.11$ & $79.72 \pm 2.49$ \\
\hline Ceniza & $3.56 \pm .28$ & $2.80 \pm 0.38$ \\
\hline Grasa total & $0.00 \pm 0.00$ & $0.02 \pm 0.02$ \\
\hline Fibra cruda & $2.50 \pm 0.00$ & $3.23 \pm .10$ \\
\hline Proteína total & $3.75 \pm 0.00$ & $1.84 \pm 0.00$ \\
\hline Carbohidratos & $12.36 \pm 1.09$ & $12.39 \pm 2.02$ \\
\hline
\end{tabular}

Tabla 2.

Características fisicoquímicas del fruto de zarzamora silvestre sp

\begin{tabular}{|l|c|c|}
\hline \multirow{2}{*}{$\begin{array}{c}\text { Característica } \\
\text { fisicoquímicas }\end{array}$} & \multicolumn{2}{|c|}{ Estados de madurez de la zarzamora silvestre } \\
\cline { 2 - 3 } & $3.01 \pm 0.07$ & Madurón \\
\hline $\mathrm{pH}$ & $6.10 \pm 0.10$ & $9.41 \pm 0.08$ \\
\hline${ }^{\circ}$ Brix & $2.92 \pm 0.02$ & $1.48 \pm 0.02$ \\
\hline $\begin{array}{l}\text { Acidez titulable (\% } \\
\text { ácido málico) }\end{array}$ & 2.09 & 6.12 \\
\hline $\begin{array}{l}\text { Índice de madurez } \\
\text { ('Brix/\% acidez) }\end{array}$ & \multicolumn{2}{|l}{} \\
\hline
\end{tabular}

Tabla 3.

Temperatura y tiempos de pasteurización del néctar a partir de la pulpa (estado pintón y maduro) y el contenido de antocianinas monómericas.

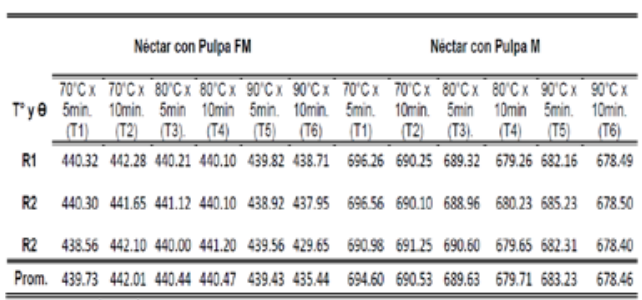

Tabla 4.

Prueba de medias (ANOVA) referido a la temperatura y tiempos de pasteurización del néctar a partir de la pulpa (estado pintón y maduro) y el contenido de antocianinas monómericas

\begin{tabular}{cclllll}
\hline & Fuente & $\begin{array}{l}\text { Suma de } \\
\text { cuadrados }\end{array}$ & Gl & $\begin{array}{l}\text { Media } \\
\text { cuadrática }\end{array}$ & F & Sig. \\
\hline Antocianinas & Inter-grupos & 73.939 & 5 & 14.788 & 3.243 & .044 \\
monomericas en & Intra-grupos & 54.714 & 12 & 4.560 & & \\
nectar con pulpa FM & Total & 128.653 & 17 & & & \\
Antocianinas & Inter-grupos & 634.911 & 5 & 126.982 & 53.573 & .000 \\
$\begin{array}{c}\text { monomericas en } \\
\text { nectar con pulpa M }\end{array}$ & Intra-grupos & 28.443 & 12 & 2.370 & & \\
\cline { 2 - 8 } & Total & 663.354 & 17 & & & \\
\hline \hline
\end{tabular}

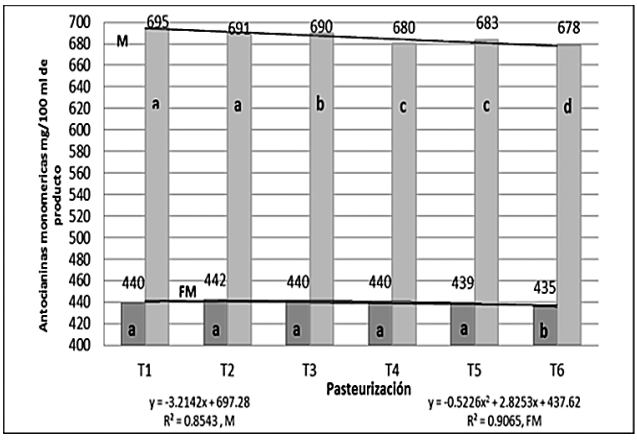

Figura 2. Representación de las medias de las puntuaciones de los diferentes tratamientos y el contenido de antocianinas monómericas en el néctar a partir de pulpa de zarzamora silvestre sp. en estado FM y M.

Tabla 5.

Temperatura y tiempos de pasteurización del néctar a partir de la pulpa (estado pintón y maduro) y el contenido de capacidad antioxidante.

\begin{tabular}{|c|c|c|c|c|c|c|c|c|c|c|c|c|}
\hline \multirow[b]{2}{*}{$T^{*} y \theta$} & \multicolumn{6}{|c|}{ Neictar con Pulpa FM } & \multicolumn{6}{|c|}{ Nectar con Pulpa M } \\
\hline & $\begin{array}{l}70^{\circ} \mathrm{Cx} \\
5 \min \\
\text { (T1) }\end{array}$ & $\begin{array}{c}70^{\circ} \mathrm{Cx} \\
10 \mathrm{~min} \\
(\mathrm{T2})\end{array}$ & $\begin{array}{l}80^{\circ} \mathrm{C} \\
x \operatorname{sinin} \\
\text { (T3). }\end{array}$ & $\begin{array}{c}8^{8} \mathrm{C} x \\
10 \mathrm{~min} \\
(\mathrm{~T} 4)\end{array}$ & $\begin{array}{l}\operatorname{soc} x \\
5 \min . \\
(T 5)\end{array}$ & $\begin{array}{c}90^{\circ} \mathrm{Cx} \\
10 \mathrm{~min} \\
\text { (T6) }\end{array}$ & $\begin{array}{l}70^{\circ} \mathrm{Cx} \\
5 \min \\
\text { (T1) }\end{array}$ & $\begin{array}{c}70^{\circ} \mathrm{Cx} \\
10 \mathrm{~min} . \\
(\mathrm{T2})\end{array}$ & $\begin{array}{l}80^{\circ} C x \\
5 \min \\
\text { (T3). }\end{array}$ & $\begin{array}{c}80^{\circ} \mathrm{Cx} \\
10 \mathrm{~min} \\
(\mathrm{~T} 4)\end{array}$ & $\begin{array}{l}90^{\prime} C x \\
5 \min \\
\text { (T5) }\end{array}$ & $\begin{array}{l}90^{\circ} \mathrm{Cx} \\
10 \mathrm{~min} . \\
\text { (T6) }\end{array}$ \\
\hline R1 & 50.00 & 48.96 & 49.28 & 46.32 & 42.16 & 38.17 & 30.50 & 29.21 & 26.65 & 25.48 & 20.10 & 18.37 \\
\hline $\mathrm{R} 2$ & 49.50 & 43.21 & 51.05 & 46.56 & 42.15 & 38.25 & 30.40 & 29.60 & 26.59 & 25.48 & 19.62 & 18.40 \\
\hline R2 & 49.90 & 46.35 & 50.23 & 46.52 & 41.25 & 38.17 & 30.50 & 28.96 & 26.89 & 25.47 & 19.22 & 18.30 \\
\hline Prom. & 49.80 & 47.84 & 50.19 & 46.47 & 41.85 & 38.20 & 30.53 & 29.26 & 26.71 & 25.48 & 19.65 & 18.36 \\
\hline
\end{tabular}

Tabla 6.

Correlación entre la capacidad antioxidante y antocianinas monómericas capacidad antioxidante del néctar a partir la pulpa en estado pintón y maduro. 


\begin{tabular}{|c|c|c|}
\hline & & $\begin{array}{l}\text { Antocianinas en néctar con } \\
\text { diferentes tratamientos (FM) }\end{array}$ \\
\hline \multirow{4}{*}{$\begin{array}{l}\text { Capacidad antioxidante } \\
\text { con diferentes } \\
\text { tratamientos (FM) }\end{array}$} & $\begin{array}{l}\text { Correlación } \\
\text { de Pearson }\end{array}$ & .795 \\
\hline & Sig. (bilateral) & .059 \\
\hline & $\mathrm{N}$ & 6 \\
\hline & & $\begin{array}{l}\text { Antocianinas en néctar con } \\
\text { diferentes tratamientos (M) }\end{array}$ \\
\hline \multirow{2}{*}{$\begin{array}{l}\text { Capacidad antioxidante } \\
\text { con diferentes } \\
\text { tratamientos (M) }\end{array}$} & $\begin{array}{l}\text { Correlación } \\
\text { de Pearson }\end{array}$ & $.831\left(^{*}\right)$ \\
\hline & Sig. (bilateral) & .041 \\
\hline
\end{tabular}

* La correlación es significante al nivel 0,05 (bilateral).

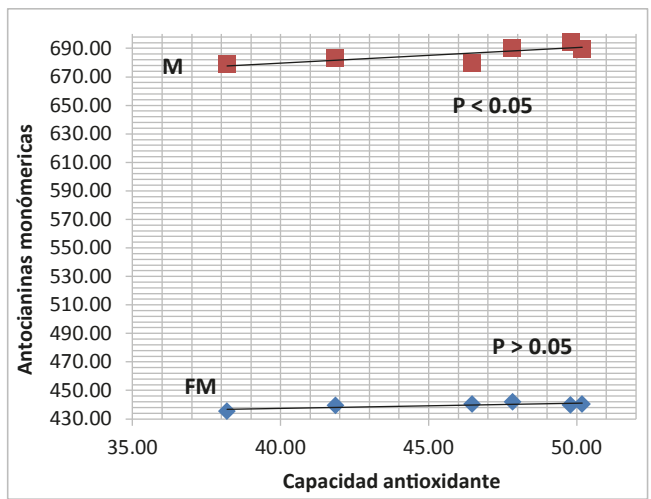

Figura 3. Representación de la correlación entre el contenido de antocianinas monómericas y capacidad antioxidante del néctar a partir de la mora silvestre $s p$. en estado FM y M

\section{Tabla 7.}

Prueba de medias (ANOVA) referido a aceptabilidad del néctar a partir de la pulpa FM

\begin{tabular}{|c|c|c|c|c|c|}
\hline Fuente & $\begin{array}{l}\text { Suma de } \\
\text { cuadrados }\end{array}$ & $\mathrm{Gl}$ & $\begin{array}{c}\text { Media } \\
\text { cuadrática }\end{array}$ & $\mathrm{F}$ & Sig. \\
\hline $\begin{array}{l}\text { Modelo } \\
\text { corregido }\end{array}$ & 35.858(a) & 6 & 5.976 & .171 & .984 \\
\hline Intersección & 13163.090 & 1 & 13163.090 & 376.170 & .000 \\
\hline Tratamiento & 3.245 & 5 & .649 & .019 & 1.000 \\
\hline Aceptabilidad & 35.858 & 1 & 35.858 & 1.025 & .314 \\
\hline Error & 3954.142 & 113 & 34.992 & & \\
\hline Total & 17220.000 & 120 & & & \\
\hline Total corregida & 3990.000 & 119 & & & \\
\hline
\end{tabular}

\section{Tabla 8.}

Prueba de medias (ANOVA) referido a aceptabilidad del néctar a partir de la pulpa $\mathrm{M}$

\begin{tabular}{|c|c|c|c|c|c|}
\hline Fuente & $\begin{array}{l}\text { Suma de } \\
\text { cuadra- } \\
\text { dos }\end{array}$ & & $\begin{array}{l}\text { Media } \\
\text { cuadrática }\end{array}$ & $\mathrm{F}$ & \\
\hline $\begin{array}{l}\text { Modelo } \\
\text { corregido }\end{array}$ & 23.800(a) & 24 & .992 & 16.625 & .000 \\
\hline Intersección & 710.533 & 1 & 710.533 & 11911.882 & .000 \\
\hline panel & 21.133 & 19 & 1.112 & 18.647 & .000 \\
\hline Trat & 2.667 & 5 & .533 & 8.941 & .000 \\
\hline Error & 5.667 & 95 & .060 & & \\
\hline Total & 740.000 & 120 & & & \\
\hline Total correg & 29.467 & 119 & & & \\
\hline
\end{tabular}

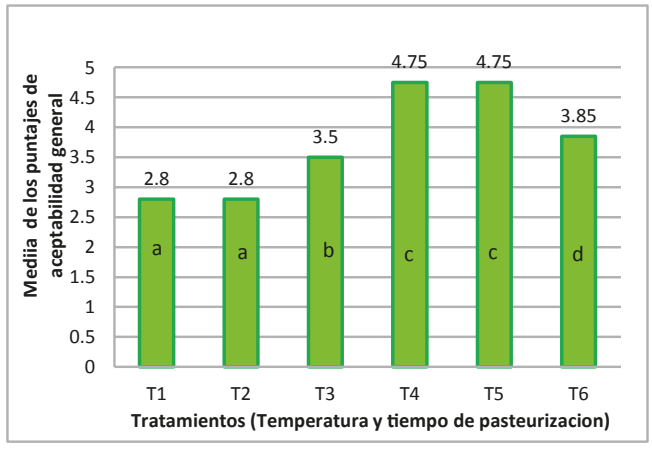

Figura 4. Representación de las medias de las puntuaciones de aceptabilidad general de los diferentes tratamientos en el néctar a partir de pulpa de mora en estado maduro.

\section{DISCUSION.}

\section{Referidos a la materia prima:}

Análisis químico proximal: De la Tabla 1. se puede apreciar que existe una diferencia notable en cuanto a proteínas, fibra y cenizas totales en comparación con el contenido de humedad, grasa y carbohidratos totales en los frutos en estados de madurez FM y M respectivamente, al respecto, Hernández y Bautista (1988), refiere que esto se debe a que los frutos están en estado de madurez Verde, Pintón y maduro, contándose días después de la síntesis que es el 
periodo de florescencia o floración de las plantas con flores; estrictamente, es el tiempo de expansión de una flor hasta que está completamente desarrollada y en estado funcional, durante el cual ocurre el proceso de polinización, si bien es frecuentemente usado para designar el período de floración en sí; el acto de florecer, esto justifica dicha similaridad en composición.

En cuanto al contenido de cenizas se tiene una disminución de $21.34 \%$ del estado FM al M esta disminuye a medida que madura la zarzamora silvestre, dichos valores son diferentes a los reportados por Montalvo (2010), que indica 3,98 g/100g de m.s. además el contenido de cenizas en base húmeda para FM es $3.56 \%$ y para el $\mathrm{M}$ es $2.8 \%$ que es mayor que lo reportado por Cabezas, (2008) que indica un valor de $0,4 \%$ dicha variabilidad es debido a los factores de crecimiento y variedad del fruto de mora.

Análisis fisicoquímico: De la tabla 2. se muestra que a medida que madura la zarzamora silvestre el `Brix e índice de madurez también aumentan, lo que indica que el fruto de mora acumula mayor cantidad de azúcares o sólidos solubles totales en la madurez (M). Rodríguez et al. (2004), menciona que hay una correlación positiva entre el estado de madurez y el aumento de los sólidos totales. Uno de los aspectos que refleja la madurez, es el comportamiento de los sólidos solubles $0{ }^{\circ}$ Brix.

Osterloh et al. (1996), indica, que el contenido de sólidos solubles está constituido por 80 a 95\% de azúcares y la medida de sólidos solubles se encuentra asociada con los azúcares disueltos en el jugo celular. Cerón (2008), en Rubus fructicosus reporta un $\mathrm{pH}$ de $3.65 \pm 0.11$ en el fruto maduro, valor muy similar al reportado.

Por otro lado García et al. (2003) Indica que los niveles de acidez expresados en acido málico varían de 2,6465 a 2,4797, sin embargo los frutos trabajados tienen entre 2.815 a 1.474 solo para el caso del fruto FM nos estaríamos acercándonos a este valor mientras que para el estado $\mathrm{M}$ tenemos valores menores a 10 reportados esto se debe principalmente a la altura sobre el nivel del mar, temperatura y tipo de suelo de Tintaypunco. También se puede observar que el valor de $\mathrm{pH}$ tiene relacion con el contenido de acidez.
Los valores del ${ }^{\circ}$ Brix especialmente en lo concerniente a la mora madura, son similares a los obtenidos por Cerón (2008), en Rubus fructicosus, 8.16 $\pm 0.28^{\circ}$ Brix. Los ${ }^{\circ}$ Brix para la mora verde y pintón muestran una tendencia también ascendente. La tendencia de la madurez fisiológica en frutas es característico un incremento en los niveles de azúcar, expresadas en este caso como sólidos solubles.

\section{Referidos al estudio de la relación de pasteurización} del néctar con pulpa de mora $s p$. (estado pintón y maduro) y el contenido de antocianinas monómericas y capacidad antioxidante.

Referidos al contenido de antocianinas monómericas: según los resultados obtenidos de la tabla 2 y tabla 3 con un $p<0.05$ se puede apreciar que existe diferencia en el contenido de antocianinas por efecto de los diferentes tratamientos de pasteurización en los néctares elaborados a partir de la pulpa de mora silvestre sp. en estado pintón y maduro.

Para el caso del néctar a partir de pulpa de mora FM puede inferir según la figura 2 que existen dos grupos el tratamiento 1, 2, 3, 4 y 5 no presentan diferencia estadística en cuanto al contenido de antocianinas, referente al tratamiento 6 se puede ver que el contenido de antocianinas se pierde en mayor proporción que los demás tratamientos.

En cuanto al néctar a partir de la pulpa en estado M se puede inferir según la figura 2, que el tratamiento 1 y 2 presentan estadísticamente el mismo contenido de antocianinas, al igual que el tratamiento 4 y 5 , el tratamiento 3 y 6 presentan diferente contenido de antocianinas. Se puede apreciar que a medida que se incrementa la temperatura y tiempo de pasteurización se pierde el contenido de antocianinas monómericas.

El néctar con pulpa FM sigue una cinética de degradación de las antocianinas de la forma polinómica con un coeficiente de determinación de 0.9065, y para el néctar con pulpa M se sigue una cinética de degradación de antocianinas de la forma lineal con un coeficiente de determinación de 0.8543 .

Hendry et al. (1992) señala la estabilidad térmica de las antocianinas varia con su estructura, pH, la presencia de oxigeno e interacciones con otros componentes del sistema. El tratamiento térmico (pasteurización) de 
Ios néctares con pulpa FM y $\mathrm{M}$ se realizó a diferentes temperaturas y tiempo $\left(70^{\circ} \mathrm{C}, 80^{\circ} \mathrm{C}\right.$ y $\left.90^{\circ} \mathrm{C}\right)$ entre 5 a 10 minutos) se controlaron adecuadamente a fin de evitar la pérdida de estos compuestos bioactivos, Markakis (1974) citado por Ojeda (2003) señala que las antocianinas son inestables cuando se exponen a altas temperaturas. Jackman y Smith (1992) mencionado por Ojeda (2003) señala que la estabilidad térmica de las antocianinas varia con la estructura, el pH, la presencia de oxígeno y las interacciones entre componentes del sistema, las características estructurales que llevan a un incremento de la estabilidad al pH también llevan un incremento a la estabilidad a la temperatura. Con algunas excepciones antocianinas sometidas a tratamientos térmicos, bajo condiciones aerobias y anaerobias.

Así mismo Calvi y Francis (1978) citado por Quispe (2003) señala que se estudió el efecto del pH en la velocidad degradativa de un numero de sistemas a temperaturas de $90^{\circ} \mathrm{C}$ e intervalos de $\mathrm{pH}$ de 1.8 a 4.0 por lo que se puede señalar que el pH influye en la degradación de antocianinas. Se debe señalar que las pérdidas en los diferentes tratamientos son mínimas ya que en algunos tratamientos no muestran diferencia significativa en la perdida de antocianinas.

Kuskoski et al. (2003), indica que las muestras ricas en antocianos son las que representan la mayor capacidad antioxidante. Definitivamente en la muestra $M$ encontramos mayor contenido de antocianinas monoméricas (tabla 4) por ello se tiene mayor poder antioxidante.

\section{Referidos a la correlación de antocianinas monómericas y la capacidad antioxidante}

Según los resultados obtenidos de la tabla 5 figura 3 Existe una buena correlación entre el contenido de antocianinas monomericas y capacidad antioxidante $(p=0.059)$ pero no significativa en los néctares elaborados con la pulpa en estado pintón., existe correlación significativa entre el contenido de antocianinas monómericas y capacidad antioxidante $\left(p<0.05 ; r=0.831^{*}\right)$ en los néctares elaborados con la pulpa en estado maduro.

Kuskoski et al. (2003), indica que las muestras ricas en antocianos son las que representan la mayor capacidad antioxidante definitivamente en la muestra
M encontramos mayor contenido de antocianinas monoméricas. La capacidad antioxidante de una sustancia no viene dada solo por la suma de capacidades antioxidantes de cada uno de sus componentes; también depende del microambiente (composición lipídica, su concentración, la temperatura, la presión de oxígeno, la presencia de otros antioxidantes, componentes habituales de los alimentos como proteínas y agua) en que se encuentra el compuesto. Los compuestos interactúan entre sí pudiendo producirse efectos sinérgicos o inhibitorios (Kuskoski et al., 2005).

Según Kähkönen y Heionan (2003) citado por Brown (2005) la malvidina es el más potente antioxidante de las antocianidinas y según Kuskoski et al. (2004) la delfinidina y cianidina tienen mayor capacidad antioxidante que la pelargonidina, malvidina peonidina y el antioxidante sintético trolox. En los dos casos aunque no tienen resultados similares, hacen referencia que las antocianidinas (malvidina, delfinidina). Por ello se puede inferir que si se encuentra una correlación alta $\left(r=0.831^{*}\right)$ entre el contenido de antocianinas monómericas y la capacidad antioxidante en los néctares elaborados a partir de la pulpa de mora del estadio maduro

\section{Referidos a la evaluación sensorial}

Según los resultados obtenidos de la tabla 6. No existe diferencia entre los tratamientos a los que se sometió en néctar a partir de la pulpa FM en cuanto a la aceptabilidad general los promedios de los puntajes a los que se calificó al producto están entre no me gusta ni me disgusta (3 puntos), de acuerdo al consolidado el color y el sabor es un atributo no adecuado para el producto elaborado a partir de la pulpa FM.

El tamaño, color y forma son características varietales. El color del fruto, cuando está inmaduro, es verde y cambia a rojo y púrpura al madurar. (Escoto, 1994). Ello influye en la coloración del producto final.

Según la tabla 7 y la Figura 4 en la evaluación sensorial del néctar a partir de la pulpa de mora madura existe diferencia estadística entre todos los tratamientos con respecto a aceptabilidad general, la mejor aceptabilidad se consigue con los tratamientos 4 y 5 que corresponden a $80^{\circ} \mathrm{C} \times 10$ min con $90^{\circ} \mathrm{C} \times 5$ min. Se debe de indicar que el tratamiento 6 disminuye 
su aceptabilidad probablemente por la pérdida de color por efecto de la temperatura.

Los pigmentos de antocianinas son relativamente inestables y la mayor estabilidad se presenta en condiciones acidas. Tanto el tono del pigmento como su estabilidad se ven influenciados por los sustituyentes en el aglicon. La degradación de antocianinas se produce no sólo durante la extracción del tejido vegetal, sino también durante el procesamiento y almacenamiento de los alimentos que las contienen (Fennema, 2000). La estabilidad de las antocianinas en los alimentos se ve notablemente afectada por la temperatura. La velocidad de degradación de la antocianinas se incrementa durante el procesamiento y almacenamiento en tanto la temperatura aumenta (Maccarone et al. 1985).

\section{CONCLUSIONES.}

En cuanto al análisis químico proximal el contenido de proteínas y cenizas totales disminuye de estadio FM a M $3.75 \%$ a $1.84 \%$ para el primero y de $3.56 \%$ a $2.8 \%$ para el segundo; con respecto a la fibra cruda ésta se incrementa de $2.5 \%$ a $3.23 \%$.

En el néctar con pulpa FM se sigue una cinética de degradación de las antocianinas por efecto de la temperaturas y tiempos de pasteurización de la forma polinómica con un coeficiente de determinación de 0.9065, y para el néctar con pulpa $M$ se sigue una cinética de degradación de antocianinas de la forma lineal con un coeficiente de determinación de 0.8543 .

Existe una buena correlación entre el contenido de antocianinas monómericas y capacidad antioxidante ( $p$ $=0.059$ ) pero no significativa; pero no significativa en los néctares elaborados con la pulpa en estado pintón, existe correlación significativa entre el contenido de antocianinas monómericas y capacidad antioxidante $\left(p<0.05 ; r=0.831^{\star}\right)$ en los néctares elaborados con la pulpa en estado maduro

En cuanto a la aceptabilidad general los promedios de los puntajes a los que se calificó al producto a partir de zarzamora sp. en estado pintón están entre no me gusta ni me disgusta (3 puntos) para los néctares a partir de la pulpa de mora en estado maduro la mejor aceptabilidad se consigue con los tratamientos 4 y 5 que corresponden a $80^{\circ} \mathrm{C} \times 10 \mathrm{~min}$ con $90^{\circ} \mathrm{C} \mathrm{x}$ 5 min. Teniendo 679.71 y $683.23 \mathrm{mg} / 100 \mathrm{ml}$ de producto de antocianinas monoméricas (Tacys) para el tratamiento 4 y 5 respectivamente, por último se tiene 25.48 y 19.65 (\% de Inhibición) DPPH de capacidad antioxidante para ambos tratamientos.

\section{REFERENCIAS BIBLIOGRÁFICAS}

- Alonso, J.; Periago, J.; Guevara, M. y Cantos, E. (2002) "Evaluación de las propiedades antioxidantes en concentrados de uva rojas y frutas rojas".

- Betelleluz, I. (2006) "Estudio de las antocianinas y evaluación de su estabilidad en extractos de mashua ((Tropaeolum tuberosum Ruiz \& Pavon)". Tesis para optar el Grado de Maestro de Magister Scientiae en Tecnología de Alimentos. Universidad Nacional Agraria La Molina. Lima - Perú.

- Cabezas Carrillo, Mercedes del Pilar. (2008) "Evaluación nutritiva y nutraceútica de la mora de castilla (Rubus glaucus.) deshidratada a tres temperaturas por el método de secado en bandejas". [Tesis Pre-grado] Escuela de Farmacia y Bioquímica. Facultad de Ciencias. Escuela Superior Politecnica de Chimborazo. Ecuador.

- Cerón, B. (2008) "Extracción, caracterización y estabilidad de antocianinas y otros compuestos antioxidantes obtenidos a partir de zarzamora" Tesis para optar el Título de Ingeniero Quimio y alimentos. Universidad de las Américas y Ciencias. Cholula, Puebla, México.

- Fennema, 0. (2000) "Química de los alimentos". Editorial Acribia. Zaragoza - España. Pp. 807-820.

- García Alonso M, de Pascual Teresa Santos Buelga C. (2004). Evaluation of the Antioxidant Properties of Fruits.

- Giusti, M., Ghanadan, H., Wrolstad, R.E. (1998) Elucidation of the structure and conformation of red radish (rapanus sativus) anthocyanins using oneand twodimensional nuclear magnetic resonance techniques. J. Agric. Food Chen.

- Hendry, G.A.F y Hougton, J.D. (1992) Natural Food Colorants. Editorial Blackie and Son Ltd. Glasgow, Escocia.

- Hernández G. R y Bautista D. (1988), Crecimiento y Cambios Bioquímicos durante el proceso de maduración de la Mora (Rubus glaucus Benth), Tesis, Facultad de Ciencias Forestales, Universidad de los Andes, Mérida, Venezuela. 
- Kuskoski E.M., Asuero A.G, Troncoso A. M., M. Carmen García M.C, Roseane F. (2004) "Actividad antioxidante de pigmentos antociánicos". Ciênc. Tecnol. Aliment., Campiñas, 24(4): 691-693.

- Kuskoski, E.M., Vega, J.M., Rios, J.J., Fett, R., Troncoso A.M. and Asuero, A.G. (2003) Characterization of anthocyanin's from the fruits of baguaçu (Eugenia umbelliflora Berg). J. Agric. Food Chem., 51, 5450-5454.

- Look, O. (1997) Antocianinas. En: Colorantes alimentarios. PUCP, Fondo Editorial $1^{\circ}$ Edición. Lima - Perú. pp 66 - 76.

- Montalvo V. D. (2010), "Evaluación de la calidad postcosecha de las accesiones seleccionadas de mora de catilla (Rubus glaucus Benth) provenientes de la provincia de Tungurahua y Bolívar) ".Proyecto de titulación previo a la obtención del título de Ingeniero Agroindustrial, Escuela Politécnica Nacional, Quito, Ecuador. pp. 58-104.

- Norma Técnica Colombiana (NTC4106). (1997). Instituto colombiano de Normas Técnicas y Certificación ICONTEC. Colombia.

- Ojeda, D. (2003) "Antocianinas totales, fenólicos totales y actividad antioxidante de las cáscaras de tres variedades de camote morado (Ipomoea batatas (L) Lam)." Tesis para optar el título de Ingeniero en Industrias Alimentarias. Universidad Nacional Agraria La Molina. Lima - Perú.

- Osterloh, A., G. Ebert, W.H. Held, H. Schulz E. and Urban. (1996). Lagerung von Obst und Südfrüchten. Verlag Ulmer, Stuttgart.253 p.

- Quispe, R. (2003) "Estudio de la extracción de antocianinas del camote morado (Ipomoea batatas (L) Lam). Tesis para optar el título de Ingeniero en Industrias Alimentarias. Universidad Nacional Agraria La Molina. Lima - Perú.

- Rodríguez, L., López, L. y García, M. (2004), Determinación de la composición química y actividad antioxidante en distintos estados de madurez de frutas de consumo habitual en Colombia, Mora, Maracuyá, Guayaba y papayuela. Facultad de Ciencias naturales, Ingeniería de Alimentos, Universidad Jorge Tadeo Lozano, Colombia.

- Wrolstad, R. (2004) Interaction of Natural Colors with other ingredients anthocyanin pigments: Bioactivity and coloring properties. Journal of Food Science Vol. 69 Nr. 5. 